the visible lineages of chloroplasts and cells. For example, the extra-nuclear determinants vary in their subordination to the nucleus probably according to their DNA or RNA constitution. Sometimes also their instability is confined to somatic tissue. This situation is a more likely analogy for antibody versatility than the instability within the nucleus which Stern discusses.

Another kind of mosaicism which seems at present to be without analogy in plants is that of the intranuclear switch. It may take several forms which Stern describes. One is the patched expression of position effects in Drosophila. These may well be the result of mere variations in the telophase placing of chromosomes with novel arrangements of euchromatin and heterochromatin. Similarly for intersexual moths. Another is the Lyon effect in mammals: the alternative condensation and inactivation of one or other of the two $X$ chromosomes (or of their differential segments) giving a mosaic appearance to heterozygous females.

The causes of these switch-actions are questionable; notably how far they have extra-nuclear components. The consequences of the mosaic $X$ effect, however, are unquestionable. What began by explaining tortoisoshell cats now extends to practical problems of human blood. For example, a woman heterozygous for haemophilia may, and one in fifty does, by random inactivation of her good $X$ leave her blood at the merey of her bad $X$. Thus she becomes classifiable as a haemophilie-putting our Mendelian definitions in some disarray.

The last of the switch-reactions, again a nuclearcytoplasmic reaction. is that leading to chromosome diminution. This property in Ascaris, Sciara, Icerya and the coccid bugs is an old story. But it now takes on a new lease of life because it leads on to elimination and also to duplication of sex chromosomes in mammals, both marsupials and rodents. It thus links up with the general problems of heterochromatin and its inactivation and of the control of the nucleus by itself. New opportunities for experiment, especially by irradiation, suddenly present themselves.

Stern's account of animal mosaics is intricate and condensed and readers will sometimes find it difficult to see the wood for the trees. He himself is more interested in analysis than synthesis. But no one could have told the story with more scrupulous accuracy. And there are few stories, as the reader will also find, in which accuracy is so properly rewarded. C. D. DaRLington

\section{HEALTH PHYSICS}

\section{Introduction to Health Physics}

By Herman Cember. (International Series of Monographs in Nuclear Energy, Vol. 105.) Pp. $x i+422$. (Pergamon: Oxford, London and New York, April 1969.) $100 s ; \$ 13.50$.

THE development of the atomic energy industry has produced a number of new branches of applied science and one of these, the physics of the health and safety aspects involved, has become known pcrhaps not altogether satisfactorily as hoalth physies. This book, one of the first of its kind, is "intended primarily as a text for a first-year course in health physies for students of environmental health science and engineering whose chief interest is radiological health".

After introductory chapters reviewing the relevant physical principles, atomic and nuclear structure, radioactivity and the interaction of radiation with matter, the author considers radiation dosimetry and the biological effects of radiation before proceeding to the details of radiation protection guides, that is, the various international authorities and their formulations regarding protection criteria. There follow chapters on instrumen- tation, external and internal radiation protection, problems associated with criticality and, finally, the evaluation of protective measures. Each chapter has appended appropriate references and a number of problems for the student.

The book has been carefully planned and is very well written. An excellent feature is the inclusion of numerous worked examples throughout the text. It is unfortunate and disappointing that so many of these contain errors. Some are typographical, several are in the powers of 10 quoted; but more serious are those in which an incorrect answer is derived and then utilized in further analysis or comment (as, for example, the figure for specific activity of ${ }^{41} \mathrm{~A}$ on page 223 , for the wall thickness on page 306 and the terminal velocity of $\mathrm{U}_{3} \mathrm{O}_{8}$ particles in example 11.1). Values of half-lives are variously quoted in the text; for example, at least four different figures appear for ${ }^{226} \mathrm{Ra}$, and the value for the atomic mass unit is likewise flexible. Fig. 10.12 (used in the examples) is printed with a faulty scale of abscissa, and the arbitrary addition of $3 \cdot 3$ HVLs in example 10.5 is likely to baffle the student, because the reference to this procedure in National Buroau of standards Handbook 76 has been omitted from Tables 10.2 and 10.3 taken from it. Equation 9.3 should state that the ionic mobility $\mu$ is for a pressure of $\mathrm{l} \mathrm{mm} \mathrm{Hg}$ to make it correct dimensionally and otherwise.

This could be a first-rate textbook and of value not only to the students for whom it is intended but to all concerned with radiological safety. Its present value, however, is largely offset by the errors of which a few examples have been mentioned. When so much thought has clearly been given to the composition of this book it is the more regrettable that equal care was not applied to checking the detailed accuracy of the text. It is to be hoped that a corrected edition may soon appear.

G. W. REeD

\section{FIRST YEAR ALGEBRA}

\section{Linear Algebra}

By A. Mary Tropper. Pp. vii + 141. (Nelson: London, May 1969.) 25s.

IN her preface the author says: "This book is an attempt to present the theory so that it can bo understood by readers who are not primarily mathematicians. Nevertheless it might be of value to those mathematics students who find transition from school to university very difficult". In fact, the book strikes me as eminently suitable for the first year mathematician, while $\mathrm{I}$ fear that the non-specialist might lose interest before completing chapter one in which groups and fields are introduced.

The material covered includes all that one would expect to find in a first year linear algebra course, with the assumption that the reader is familiar with matrix manipulation and with determinants. I would have preferred to see these topies included, but I have few other complaints; the book is easy to read, being written in a clear and precise style and being printed in a most agreeable format. Plenty of illustrative examples are included in the text and each chapter includes problems for the reader. I noticed only one misprint (in line 2 of page 15). It would perhaps have been a help if there had been an index of symbols because I am sure that the reader, particularly the non-specialist, might soon forget the meanings of $Q$ (the rationals) and $Z$ (the integers), for example.

This book is very good value for money and should be within the reach of students' pockets. It is perhaps a pity that the cover is not more firmly attached. Mine came off before $I$ reached page 30 .
J. K. REID 